\title{
Empiric antifungal coverage of the septic cirrhotic: A case report of Acinetobacter baumanii with concomitant fungal infection in a new cirrhotic
}

\author{
Zachary Lazev ${ }^{1}$, Nandakumar Mohan ${ }^{1}$, and Bhavna Sharma ${ }^{1}$ \\ ${ }^{1}$ Albert Einstein Medical Center
}

February 7, 2022

\begin{abstract}
Acinetobacter baumannii is an aerobic gram-negative bacillus and nosocomial pathogen that predominantly affects individuals in the ICU. We present a case of a patient without any known medical problems or recent hospitalizations presenting with Acinetobacter baumannii bacteremia and a concomitant spontaneous fungal peritonitis in the setting of newly diagnosed cirrhosis
\end{abstract}

\section{Title Page:}

Empiric antifungal coverage of the septic cirrhotic: A case report of Acinetobacter baumanii with concomitant fungal infection in a new cirrhotic

\section{Informed Consent:}

Due the patient's death, written informed consent was obtained via next of kin from the patient to publish this report in accordance with the journal's patient consent policy.

Case History/Examination:

Acinetobacter baumannii is an aerobic gram-negative bacillus and nosocomial pathogen that predominantly affects individuals in the intensive care unit. We present a case of a patient without any known medical problems or recent hospitalizations presenting withAcinetobacter baumannii bacteremia and a concomitant spontaneous fungal peritonitis in the setting of newly diagnosed cirrhosis.

Our patient had no known medical history other than alcohol abuse. The patient developed hepatic encephalopathy and subsequent aspiration pneumonia resulting in Acinetobacter baumannii bacteremia from pneumonia. Acinetobacter baumannii is especially known for its multidrug resistance (MDR). This case was further complicated by concomitant fungal peritonitis. Cirrhotic patients can have cirrhosis-associated immune dysfunction (CAID), leading to an immunosuppressed state. Furthermore, cirrhotic have physiologic changes such as a chronic vasodilatory state with hyperdynamic circulation and alterations in thermoregulation, that may independently make patients more susceptible and mask underlying sepsis. Therefore, identification of sepsis can be intrinsically challenging in the cirrhotic patient. Early and broad anti-microbial coverage has been a well proven way to improve mortality and lead to better clinical outcomes for critically ill patients, but the question arises, are there times where cirrhotic patients may need even broader coverage? Our case report explores these challenges and presents questions requiring further research in this patient population.

Introduction: 
There is a well-established connection that cirrhosis has a negative outcome on a patient's health. Patients with cirrhosis often have complications including, but not limited to portal hypertension, splenomegaly, increased risk of bleeding, risk of encephalopathy among other complications which lead to increases in morbidity and mortality ${ }^{1}$. Although these complications are well established, cirrhosis-associated immune dysfunction (CAID) is not as often considered in the setting of the septic patient. Sepsis and septic shock can be identified by hallmarks such as, temperature, hemodynamic instability, heart rate and leukocytosis. Physiologic changes of cirrhosis can compromise the identification of septic shock. Patients with cirrhosis live in a vasodilatory state secondary to nitric oxide release from the cirrhotic liver ${ }^{2}$. Furthermore, patients are in a hyperdynamic state that can alter thermoregulation ${ }^{3}$. Sequestration of blood cells in patients with cirrhosis and associated portal hypertension can cause leukopenia in the setting of spleen sequestration ${ }^{4}$.

CAID predisposes patients to diminished levels of innate and acquired immunity ${ }^{5}$. Septic shock associated with bloodstream infections can occur in up to $21 \%$ on cirrhotic patients, ten-fold higher than patients without cirrhosis ${ }^{6}$. Indicators of severe infection can be masked in a patient due to the altered physiology of cirrhosis. Hemodynamic instability, altered thermoregulation, and skewed white blood cell counts in the septic patient can lead to a critical clinical delay in the identification of the septic patient.

Acinetobacter baumannii is a gram-negative bacillus that is known as an opportunistic infection, typically found in patients with prolonged hospital stays ${ }^{7,8}$. Acinetobacter baumannii often colonizes the oropharynx, and is well-established as a MDR organism ${ }^{9}$. Cirrhotic patients with CAID infected with a MDR organism such as Acinetobacter baumannii have increased rates mortality ${ }^{10}$.

Our case is a patient with suspected cirrhosis with no significant medical history but with a history of extensive alcohol consumption. The peculiarity of this case was the self-isolation status of the patient from the Covid-19 pandemic. Family stated the family had not left the house for nearly two years due to concern of Covid-19 exposure. This case provokes the question; how this patient acquired such a rare bacterial infection with no predisposing history or exposure. This case highlights the natural physiologic changes due to cirrhosis and shows that physicians need to stay vigilant in anticipating severe sepsis from rare organisms in this high-risk patient population.

Case Description:

The patient is a 40-year-old gentleman without significant past medical history who presented to the hospital for worsening shortness of breath, abdominal distension and lower extremity swelling over the past few days. Patient had extensive history of alcohol use but does not have confirmed diagnosis of cirrhosis.

Physical exam was significant for and abdominal exam with a distended abdomen. Respiratory exam was significant for lung sounds diminished bilaterally, worse in the left lower lobe. Cardiovascular exam significant for tachycardia. Neurological exam positive for altered mental status not alert and oriented to person, time or place which is normal at baseline. Patient had initial hypotension with BP $75 / 45$, other vital signs of respiratory rate of 34 , heart rate 150 , temperature 36.5 , saturating $83 \%$ on room air. Patient had a diagnostic and therapeutic paracentesis was also done $(2.3 \mathrm{~L})$, with paracentesis labs not showing any SBP. Patient required intubation for persistent altered mental status with concerns for maintenance of airway. Initial labs were significant for hyponatremia, hypokalemia, hypomagnesemia, high anion gap metabolic acidosis primarily driven by lactic acidosis, acute kidney injury, direct bilirubinemia with normal alkaline phosphatase (hypoalbuminemia with INR of 2.2; AST 187/ALT 15), hypoglycemia, leukocytosis with neutrophilic predominance, anemia with hemoglobin of 12.7. Chest x-ray was significant for reticular and airspace opacities in the right middle and lower lobe with left-sided pleural effusion. CT of the abdomen and pelvis was done which showed right lower lobe consolidation, with large left sided pleural effusion and ascites. Patient was started on vaso-pressors. Given 1 dose of piperacillin-tazobactam was also given for concern of septic shock on initial admission. Thiamine $100 \mathrm{mg}$ intravenously was also given.

Blood cultures were drawn before antibiotics were given, which resulted positive for Acinetobacter baumannii in the blood. Suspected source due to chest x-ray was pneumonia. Antibiotics were escalated to Vancomycin and Cefepime from piperacillin-tazobactam before blood cultures resulted. Cefepime was 
escalated to Meropenem once cultures resulted positive for Acinetobacter baumannii infection. Initially, the patients' vaso-pressor requirements decreased as norepinephrine was titrated down on vancomycin and meropenem. Antibiotics were deescalated to Unasyn as sensitives resulted forAcinetobacter baumannii infection. Yeast grew a few days after fluid was collected, micafungin was started. Within the next 24 hours, the patient's vaso-pressor requirement increased from norepinephrine to additional vasopressin and phenylephrine for blood pressure support. Furthermore, epinephrine was added as a fourth pressor as the patient required CRRT due to AKI. Patient became anuric, was started on CRRT and the patient succumbed to the septic shock secondary to theAcinetobacter baumannii infection and the peritoneal yeast infection.

\section{Discussion:}

This case presentation provokes multiple questions most importantly, but not limited to, the timing of antibiotics used in the hospital setting. Also, the consideration for empiric antibiotics for bacteremia and possible fungal infections in the cirrhotic. Interestingly, the connection between spontaneous bacterial peritonitis (SBP) and the prophylactic use of ciprofloxacin is well established, although the necessity to empirically cover for fungus in the cirrhotic septic patient is not ${ }^{11}$. Of note, other immunosuppressive conditions such as HIV are treated with prophylactic medications to prevent serious infections, including fungal infections. A retrospective study by Kumar et al. suggests that every hour of delay regarding antibiotic coverage in a cirrhotic lead to decreased survival by $7.6 \%{ }^{12}$. Cirrhosis alters normal physiology, often masking indicators of severe sepsis such as hypotension, leukopenia, and dysfunction of thermoregulation ${ }^{2,3,4}$. Thus, early detection of severe sepsis and appropriate coverage can be delayed, further leading to difficult detection

Plessier et al. established fungal infections are responsible for up to $15 \%$ of cirrhotic infections ${ }^{13}$. The patient presented in this case was not started on fungal coverage until multiple days into the hospital admission. During the hospital course the patient initially had decreasing pressor requirements attributed to appropriate bacterial coverage for Acinetobacter Baumanii as well as other unknown microbes with vancomycin and meropenem, but shortly after fungi grew in the collected peritoneal fluid. Despite subsequent micafungin coverage, the patient went into rapid hemodynamic decompensation, multiorgan system failure and ultimately succumbed to his condition. This raises the question: Do patients with cirrhosis requiring intensive levels of critical care benefit from empiric antifungal coverage?

Hassan et al. highlights that delayed treatment and diagnosis can become fatal and although fungal infections are rare, empirically treating for fungal infections can improve the outcome ${ }^{14}$. Retrospective analysis for the management of this case, the suspected result of decompensation would be attributed to a lack of fungal empiric coverage on arrival to the intensive care unit. This case provokes multiple questions. Primarily, when is it appropriate to provide antifungal coverage in a patient? Maertenset al. outlines the use for empiric antibiotic coverage for antifungals who have persistent sepsis for 4-7 days after broad spectrum antibiotics ${ }^{15}$. Based on these recommendations, the patient was appropriately treated within those parameters. This study specifically assesses antifungal requirements in a neutropenic and cirrhotic patient. Antifungal coverage in the septic cirrhotic is not well established. Do patients with refractory septic shock secondary to broad spectrum or appropriate sensitive coverage warrant immediate antifungal consideration? Furthermore, should cirrhosis be treated as an immunosuppressive condition and warrant greater initial empiric coverage? These questions are poorly understood but this case highlights the importance and the necessity to explore these questions to improve patients' outcomes.

The other peculiar aspect of this case is the sheer fact that a patient with no underlying exposure could present with Acinetobacter baumanii bacteremia suspected from pneumonia. The patient had no underlying past medical history except for newly diagnosed cirrhosis on the admission. The patient was self-isolated due to the Covid-19 pandemic which makes this finding even more profound. Dijkshoornet al, outlines that Acinetobacter baumanii is a rare infection often multi-drug resistant (MDR) and often associated with ventilator associated pneumonia (VAP $)^{16}$. This case illustrates cirrhosis as the only underlying known immunosuppressive condition in a patient with a rare typically nosocomial acquired infection complicated by fungal peritonitis with no previous hospitalization or exposure. Cirrhosis evidently may have a profound impact on a patients' immune system due to CAID. Therefore, this transient level of natural immunosuppres- 
sion in the cirrhotic population should strongly be considered in the intensive care setting when determining appropriate treatment.

\section{Conclusion:}

Severe sepsis is a critical diagnosis and often seen in the intensive care unit. Sepsis can be identified by different hallmarks which can often be skewed by the altered physiology of the cirrhotic patient. Ultimately, cirrhosis not only masks signs of sepsis but can predispose patients to worse outcomes due to the immune susceptibility of (CAID). Our case highlights the profound impact cirrhosis can have on a patient with sepsis. Moreover, the case suggests that further research is needed to elucidate appropriate antibiotic and antifungal considerations in empiric treatment for the septic cirrhotic. Further exploration of such information may improve mortality rates in this patient population.

Author Contributions:

Zachary Lazev, DO. Author Contributions: First author, wrote the case, was the intern resident with this patient

Nandakumar Mohan, DO. Author Contributions: Second author, edited the case, assisted in areas, was the senior resident with this patient

Bhavana Sharma, MD. Author Contributions: Attending Physician provided recommendations and supported the submission of the case

\section{References:}

1 Nusrat, S., Khan, M. S., Fazili, J., \& Madhoun, M. F. (2014). Cirrhosis and its complications: evidence based treatment. World journal of gastroenterology , 20 (18), 5442-5460. https://doi.org/10.3748/wjg.v20.i18.5442

2 Martell, M., Coll, M., Ezkurdia, N., Raurell, I., \& Genescà, J. (2010). Physiopathology of splanchnic vasodilation in portal hypertension. World journal of hepatology , 2 (6), 208-220.

https://doi.org/10.4254/wjh.v2.i6.208

3 Mani, A. R., Mazloom, R., Haddadian, Z., \& Montagnese, S. (2018). Body temperature fluctuation analysis in cirrhosis. Liver international : official journal of the International Association for the Study of the Liver , 38 (2), 378-379. https://doi.org/10.1111/liv.13539

4 Qamar, A. A., \& Grace, N. D. (2009). Abnormal hematological indices in cirrhosis. Canadian journal of gastroenterology = Journal canadien de gastroenterologie , 23 (6), 441-445. https://doi.org/10.1155/2009/591317

5 Noor, M. T., \& Manoria, P. (2017). Immune Dysfunction in Cirrhosis. Journal of clinical and translational hepatology , 5 (1), 50-58. https://doi.org/10.14218/JCTH.2016.00056

6 Bartoletti, M., Giannella, M., Caraceni, P., Domenicali, M., Ambretti, S., Tedeschi, S., Verucchi, G., Badia, L., Lewis, R. E., Bernardi, M., \& Viale, P. (2014). Epidemiology and outcomes of bloodstream infection in patients with cirrhosis. Journal of hepatology , 61 (1), 51-58.

7 Howard, A., O’Donoghue, M., Feeney, A., \& Sleator, R. D. (2012). Acinetobacter baumannii: an emerging opportunistic pathogen. Virulence , 3 (3), 243-250. https://doi.org/10.4161/viru.19700

8 Montefour, K., Frieden, J., Hurst, S., Helmich, C., Headley, D., Martin, M., \& Boyle, D. A. (2008). Acinetobacter baumannii: an emerging multidrug-resistant pathogen in critical care. Critical care nurse , 28 (1), 15-26.

9 Sebeny, P. J., Riddle, M. S., \& Petersen, K. (2008). Acinetobacter baumannii skin and soft-tissue infection associated with war trauma. Clinical infectious diseases : an official publication of the Infectious Diseases Society of America , 47 (4), 444-449. https://doi.org/10.1086/590568 
10 Ekpanyapong, S., \& Reddy, K. R. (2019). Infections in Cirrhosis. Current treatment options in gastroenterology , 17 (2), 254-270. https://doi.org/10.1007/s11938-019-00229-2

11 Téllez-Ávila, F., Sifuentes-Osornio, J., Barbero-Becerra, V., Franco-Guzmán, A., Ruiz-Cordero, R., AlfaroLara, R., Hernández-Ramírez, A., \& Vargas-Vorácková, F. (2013). Primary prophylaxis with ciprofloxacin in cirrhotic patients with ascites: a randomized, double blind study. Annals of hepatology , 13 (1), 65-74.

12 Kumar, A., Roberts, D., Wood, K. E., Light, B., Parrillo, J. E., Sharma, S., Suppes, R., Feinstein, D., Zanotti, S., Taiberg, L., Gurka, D., Kumar, A., \& Cheang, M. (2006). Duration of hypotension before initiation of effective antimicrobial therapy is the critical

13 Plessier, A., Denninger, M. H., Consigny, Y., Pessione, F., Francoz, C., Durand, F., Francque, S., Bezeaud, A., Chauvelot-Moachon, L., Lebrec, D., Valla, D. C., \& Moreau, R. (2003). Coagulation disorders in patients with cirrhosis and severe sepsis. Liver international : official journal of the International Association for the Study of the Liver , 23 (6), 440-448. https://doi.org/10.1111/j.1478-3231.2003.00870.

14 Hassan, E. A., Abd El-Rehim, A. S., Hassany, S. M., Ahmed, A. O., Elsherbiny, N. M., \& Mohammed, M. H. (2014). Fungal infection in patients with end-stage liver disease: low frequency or low index of suspicion. International journal of infectious diseases : IJID : official publication of the International Society for Infectious Diseases , 23 , 69-74. https://doi.org/10.1016/j.ijid.2013.12.014

15 Maertens, J., Marchetti, O., Herbrecht, R., Cornely, O. A., Flückiger, U., Frêre, P., Gachot, B., Heinz, W. J., Lass-Flörl, C., Ribaud, P., Thiebaut, A., Cordonnier, C., \& Third European Conference on Infections in Leukemia (2011). European guidelines for antifungal management in leukemia and hematopoietic stem cell transplant recipients: summary of the ECIL 3-2009 update. Bone marrow transplantation , 46 (5), 709-718. https://doi.org/10.1038/bmt.2010.175

16 Dijkshoorn, L., Nemec, A., \& Seifert, H. (2007). An increasing threat in hospitals: multidrug-resistant Acinetobacter baumannii. Nature reviews. Microbiology , 5 (12), 939-951. https://doi.org/10.1038/nrmicro1789 